\title{
Health Folder/Osasun Karpeta
}

\section{Dabi Santano Garcia*}

Osakidetza Directorate General, ES, Spain

*Corresponding Author: Dabi Santano Garcia, Osakidetza Directorate General, ES, Spain.

Received: August 30, 2019; Published: September 10, 2019

In 2010, within the framework of the Osarean Project "Osakidetza no in-person", the idea of Health Folder emerged in order to develop non-face-to-face channels between citizens, patients and Osakidetza.

Osarean was set up as a multi-channel platform based on the ITCs (Information and Communication Technologies) tools and which has as its mission to develop a relationship and service model focused on the needs of patients and citizens who, among others, enhance the management of the prevention, promotion and maintenance of health and introduce the culture of co-responsibility and self-care.

A European project called "Sustains" was used to continue its development.

Health folder?? allows the patient to access their clinical history, enabling in turn new non-face-to-face communication channels with professionals, and allowing to enrich the clinical history by the program itself patient.

From the beginning, different professionals with different profiles have been the ones who have been part of the Osarean team; administrative, COMPUTER, health services,...

The diversity of opinions of all of them has helped us to evolve the folder day by day, including progressively new services and functionalities.

The team is constantly in contact with the citizens, as their suggestions also help us, and much, to improve.

\section{Objectives}

The objectives of Folder are as follows

- $\quad$ Enable citizens to access, through the Osakidetza portal (computer, tablet, mobile phone), to the information of their medical history in a secure and confidential way, at any time and from anywhere.

- $\quad$ Facilitate citizens' non-face relationship with Osakidetza professionals.

- $\quad$ To enhance the role of the citizen as responsible for the promotion of his health.

- $\quad$ To help prepare the Basque health system for the needs of the future, contributing to its sustainability.

Access

The Health Folder is a service that all citizens of the Basque Country have who have TIS. (Sanitary Individual Card ???).

There are currently 2 access modes

- $\quad$ By digital certificate, that is, an electronic card such as the DNIe, the Municipal Citizen Card or other professional cards. By this means it is necessary to have a card reader on the computer, but since we do not all have it other methods have been developed.

- $\quad$ By FEA, i.e. an Advanced Electronic Signature. It's called the B@kQ or BakQ and it's the equivalent of a ship game along with its reference number or ID and a personal key. This way you don't need to enter any card so you can enter your health folder from any device whether it's a Tablet, Smartphone,...

- $\quad$ Both accesses guarantee the security and confidentiality of the data and is that we must not forget that we are dealing with health information which requires a high level of security. 


\section{Services}

Health Folder services can be grouped into 3 blocks

Those that allow you to consult and retrieve information

- $\quad$ Affiliation data: basic patient data is displayed along with the assigned healthcare professional. In addition fields are displayed that the user can update as well as phone and email.

- My medical history: Include discharge, primary, lab, radiological tests, surgical. In addition to the vaccination history, dosimetric (radiation received according to the TACs performed),active treatment sheet (from where the Sintrom dosing schedule can be accessed if taken). There is also the possibility to download a QR code with a summary of the medical history.

- Upcoming appointments: Patient can check the appointments you have both in Primary as a Hospital as well as downloading a reminder of it that is integrated into the device's calendar ore mail it to you.

- $\quad$ Surgical waiting list: Once included in the waiting list, information is displayed. Patient Service information can also be requested via email from the same window.

Those that allow to enrich the medical history

- $\quad$ Self-tracking programs: Tobacco and alcohol use, weight control and blood pressure.

- Uploading documents: The patient himself can incorporate health reports into his own medical history after the validation of the healthcare professional. This way professionals do not have to enter such information.

Those that allow you to interact with the healthcare system

\section{Patient's diary}

Is a one-way channel through which the patient can record information about their health status at the request of healthcare professionals, and this is automatically included in their own medical history.

In this way the patient can record daily how he is and if there are any conditions that influence his condition at that time. The healthcare professional may track the patient's condition according to the information recorded or through forms.

\section{Messages to the patient}

It is another one-way channel but unlike the previous one it is the professional who contacts the patient to send him information, recommendations and adjustments in the treatment for example.

The patient receives an email notifying them that they have a new message in their health folder. After entering your folder you will be able to see this message.

Through this tool we can achieve a closer relationship with the patient and transmit the feeling and reality that we closely follow their process and situation, updating the information that is necessary.

Doubts with your doctor

Is a two-way non-face-to-face care channel, that is, there is an interrelationship between the patient and the professional himself.

The professional must authorize the patient the option to resolve their doubts Through the Health Folder for primary and/or specialized.

The patient enters his doubt and the professional accessing the virtual appointment visualizes the data.

Once the professional's response is sent, the patient receives an emitted response co-signature emailand must enter their Health Folder to view it.

\section{New services}

Access to other people

The Osakidetza Health Folder service has intended, in the first phase, to provide only the owner of the same, over 16 years of age, with access to his assistance and administrative information by electronic means.

Given the diversity of request for access by duly accredited and authorized. The same, a new functionality has been developed that allows these accesses from the Health Folder itself.

Taking into account Law 41/2002, of November 14,Basic regulatory of patient autonomy and rights and obligations in the field of information and clinical documentation, Decree 38/2012, of March 13 ,onhistory clinical and rights and obligations of patients and 
health professionals in matters of clinical documentation, and El Report 0339/2015, of October 8 of the Legal Cabinet of the Spanish Data Protection Agency and guarantees access to the third-party $\mathrm{HC}$ in the following cases:

- $\quad$ Parents and Guardians (provided parents have parental authority or guardianship document as appropriate).

- $\quad$ Representatives of legally incapacitated patients (with the corresponding document).

- $\quad$ People older is of age to authorize it.

Access to the newborn's folder

Parents who wish to do so can manage this procedure in the Hospital itself and thus when the TIS of the newborn is final they can access the health folder of their children.

\section{Sintrom dosing schedule}

Currently any patient receiving Sintrom can access the sheet through his Health Folder with the latest daily dosing regimen of the Sintrom being able to view, download or print it.

Data

As you can see in the attached graphic, the number of accesses to Health folder been almost doubled annually, exceeding 550,000 last year.

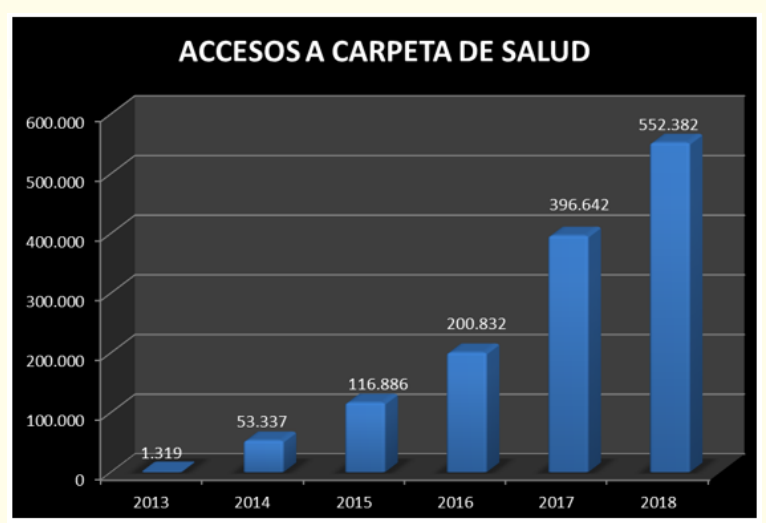

Figure 1

If we focus on the number of different users using Health Folder, we also see how the number has been growing year after year exceeding 50,000last year.

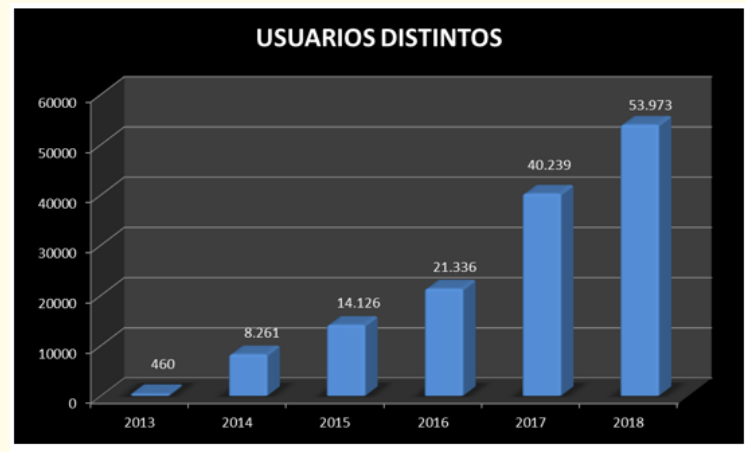

Figure 2

\section{Conclusions}

The incorporation of new technologies into the healthcare model

- It contributes to facilitating the objective of health integration,

- It supports collaboration between different actors and healthcare levels within the health system.

- It gives healthcare professionals a comprehensive view of patient information and situation.

- Enables new channels of communication and interaction with the patient.

Volume 3 Issue 10 October 2019

(C) All rights are reserved by Dabi Santano Garcia. 